\title{
Role of Micronutrients on Type Ii Diabetes Mellitus
}

\section{P Rajalakshmy*}

Lecturer in Nutrition, Kasturba Gandhi Nursing College, India

*Corresponding Author: P Rajalakshmy, Lecturer in Nutrition, Kasturba Gandhi Nursing College, India.

Received: July 31, 2019; Published: September 10, 2019

\begin{abstract}
The Diabetic and pre- diabetic population increases globally due to the population over growth, aging, urbanization the major reasons are unhealthy eating habits, increasing prevalence of obesity and physical inactivity. Type II Diabetes Mellitus is one of the most widely spread and severe disorder. Its currently being the fourth mortality reason. Type 2 DM is a metabolic disorder, with hyperglycemia as the dominant feature, as a result of reduced secretion of insulin and increased insulin resistance. Globally Diabetes mellitus is a chronic metabolic disorder associated with the increased free radical production leading to oxidative damage, and Previous studies indicated that marginal Micronutrient deficiency is more prevalent among diabetic adults, compared to the normal adult population. However, most of the micronutrients are involving in much way either as part of the etiology or effect of this chronic pathology. The DM complication increases in case of imbalance between free radical formation and their control by natural antioxidants. Thus, antioxidant property of micro nutrients is very important in the development of the disease and its complications, while other non-antioxidant micro nutrients have also shown some relationship.
\end{abstract}

Keywords: Diabetes Mellitus; Micro Nutrients; Antioxidants; Supplement.

\section{Introduction}

Worldwide, Diabetes mellitus is a leading cause of morbidity and mortality, It is also associated with life threatening and potentially disabling macro- and micro-vascular complications [1]. The worldwide prevalence of Type II Diabetes Mellitus is increasing steadily [2]. Approximately, 150 million people worldwide are affected by Type II Diabetes Mellitus and this integer is expected to double in the next 20 years [3]. The DM suffering patients was reported to be over 200 million people globally, $90 \%$ of those have Type II Diabetes Mellitus, characterized by insulin resistance, hyper insulinaemia, $\beta$-cell dysfunction and subsequent $\beta$-cell failure [4].

\section{Anti oxidant on type ii diabetes mellitus}

The administration of antioxidants such as Zinc, Magnesium, Selenium, Vitamin A, Vitamin-C and vitamin E may improve the tissue response to insulin and increase the efficacy of drugs. The major reason for this pathogenesis of diabetes and its' complications are Oxidative stress [5].
Micro nutrients on type ii diabetes mellitus

Since, Type II DM can be stated as metabolic abnormality So, the Micro Nutrients as a component of enzymes may be playing an important role in development and control of Type II DM.Insulin action on reducing blood glucose was reported to be potentiated by many trace elements which mentioned in the following [6].

Zinc

Recent study in a decade's indicated that marginal zinc deficiency is more prevalent among Type II DM adults, compared to the normal adult population [7]. Abnormal zinc and lipid plasma levels occur more frequently in metabolically uncontrolled DM patients. When glucose entered into the blood stream the stored insulin from the pancreas which binds with the zinc will get realized. It has its anti-inflammatory effects where its play a role in abolishing inflammatory markers such as C-reactive proteins. Zinc also helps to get rid of substances that cause inflammation in cells, helps to preserve cell health and insulin sensitivity [8]. 


\section{Copper}

Copper is an essential trace mineral being a co-factor for many enzymes. Copper involved in SOD activity. Type II Diabetes Mellitus associated with increased free radical production leading to oxidative damage, and many of the pathological effects of copper overload are consistent with an oxidative damage to membranes. Once again it is proved that, if there is a misbalance in the copper ions among Type II DM increases their complications [9].

\section{Chromium}

Trivalent chromium ( $\mathrm{Cr}$ ) is an essential trace mineral in metabolism of carbohydrate, lipid and protein. So, currently it has been prescribed by some physicians in an intention to control Type II DM. Many studies have shown that $\mathrm{Cr}$ could light up insulin receptors also stimulate the liver enzyme glucokinase, and enhance pancreatic $\beta$ islets. $\mathrm{Cr}$ can control the hyperglycemia in type 2 DM [10].

\section{Magnesium}

Magnesium is an essential mineral involved in glucose homeostasis. It is a cofactor for various enzymes in carbohydrate metabolism. It is also involved at multiple levels in insulin secretion, binding and activity. Reduced level of magnesium has been documented in Type II DM. lower level of serum magnesium will result in glucose homeostasis and insulin sensitivity among Type 2 DM patients [11].

\section{Vitamin- A}

It is stated that DM affected population will suffer from lower level of plasma vitamin A and carotenoids. A study proved that increased level of $\beta$ carotene being associated with lower risk of Diabetes Mellitus [12].

\section{Vitamin- B1}

Thiamine (B1) play many role in the body like act as a coenzyme in the active transference of aldehyde groups and glycation, it is also play as neuro-transmission and neuronal conductivity, lower level of this thiamine may leads to various diabetic complication. Low levels of thiamine and increased renal excretion have found in both Type 1 and type 2 diabetic patients [13].

\section{Vitamin- B6}

Pyridoxal-5'-phospate (PLP) is an active form of vitamin B6. This vitamin $\mathrm{B} 6$ will It is an amino transferase, also acting as a co- enzyme for glucose-phosphorilase for the utilization of glycogen in liver and muscle, thus participating actively in glucose metabolism [14].

\section{Vitamin- B3}

NAD and NADH has nicotic acid as an important compound, this plays a major role in ATP production and energy efficiency at the cellular level. supplementing niacin towards DM patients will reduce the monocyte adhesion to endothelial cells [15].

\section{Vitamin- B7}

A study declared that biotin supplementation Type II DM rats ended with anti-diabetic effects, apparently preventing insulin resistance in skeletal muscle by an increase in the expression of the glucose transporter protein GLUT4 [16].

\section{Vitamin- B12}

Type II Diabetes Mellitus is an oxidative stress disease; vitamin $\mathrm{B}_{12}$ and folic acid deficiencies in a diabetic patients have been found associated to oxidative stress, in relation to a resulting hyperhomocysteinemia [17] As a result of this association, it is conceivable that vitamin $B_{12}$ deficiency should be considered a risk factor for diabetic complications.

\section{Vitamin- B9}

The Glycosylated Hemoglobin Fasting Blood Glucose got reduces with the supplementation of Folate and also improved the glycemic control, Serum Insulin and Insulin Resistance as well as homocysteinemia in Type II Diabetes Mellitus patients. The pyridoxine, folate and vitamin $\mathrm{B}_{12}$ supplementation shows Positive effects on signs and symptoms of diabetic retinopathy [18].

\section{Vitamin- C}

Studies have observed that Type II Diabetes Mellitus patients have lower circulating levels of vitamin $\mathrm{C}$ and higher levels of oxidative stress markers compared to a non- diabetic patients. Supplementation studies with Vit-C showed the diverging results on plasma concentration of antioxidants [19].

\section{Vitamin- D}

Vitamin D deficiency is a risk factor for cardiovascular disease in Type II Diabetes Mellitus patients. Elderly patients with type II DM are prone to develop vitamin D deficiency, which is very common in European countries, associated to inadequate intake, low sunexposure and age-related alterations in absorption, synthesis and metabolism of the vitamin $\mathrm{D}[20]$. 
Vitamin- E

The effect of antioxidant properties in the vitamin E helps in reducing the risk of diabetes and its complications; a decrease in plasma tocopherol has been observed in diabetic subjects with longer duration of the disease. Few study proved that supplementation with vitamin $\mathrm{E}$ have shown positive effects on blood glucose on Type II Diabetes Mellitus [21].

\section{Conclusion}

Even though Micronutrients exert important effects on risk of Type II Diabetes Mellitus as well as its progression and complications, there is no enough evidence in most cases to suggest micronutrients supplementation in the general diabetic population. In this sense, it is necessary to undertake dietary evaluations to identify specific intake deficiencies and establish recommendations. The use of supplements carries the risk of toxicity However, there is enough scientific evidence to recommend the supplementation of Micronutrients in those patients with Type II Diabetes Mellitus, who are being treated with Metformin, to reduce the risk of developing neuropathy and its consequences.

\section{Bibliography}

1. Levin M and Pfeifer M. Uncomplicated Guide to Diabetes Complications. 3rd edition. Alexandria, Virginia: American Diabetes Association (2009).

2. Freeman HRD. Cox. Type-2 diabetes: A cocktail of genetic discovery. Human Molecular Genetics 15 (2006): R202-209.

3. Afkhami-Ardekani M., et al. "Epidemiological survey of NIDDM inpersons over 30 years old in Yazd province". Shahid Sadoughi University of Medical Sciences and Health Services 9 (2001): 22-27.

4. Stumvoll M., et al. "Type 2 diabetes: principles of pathogenesis and therapy". Lancet 365 (2005): 1333-1346.

5. Kelly F. "Use of antioxidants in the prevention and treatment of disease". Journal of the International Federation of Clinical Chemistry 10 (1998): 21-23.

6. Candilish DJ. "Minerals". Journal of the American College of Nutrition 17 (2000): 286-310.

7. Lee JH., et al. "Zinc and copper status of middle- and old-aged woman in type 2 diabetes". The Korean Journal of Nutrition 38 (2005): 56-66
8. Ortega R., et al. "Poor Zinc Status is Associated with Increased Risk of Insulin Resistance in Spanish Children". British Journal of Nutrition 107 (2012): 398-404.

9. Lefevre M., et al. "Copper deficiency- induced hypercholesterolemia: effects on HDL subfractions and hepaticlipoprotein receptor activity in the rat". Journal of Nutrition 116 (1986): 1735-1746.

10. Cefalu WT and Hu FB. "Role of chromium in humanhealth and in diabetes". Diabetes Care 27.11 (2004): 2741-2751.

11. Vincent JB. "Beneficial effects of chromium (III) andvanadium supplements in diabetes". Nutrition TherapInterv Diabetes Metabolism Syndrome (2012): 381-391.

12. F Hussain., et al. "Trace elements status in type 2 diabetes". Bangladesh Journal of Medical Science 8 (2009): 44-45.

13. Gavrilov V., et al. "Kidney function and retinol status in type 2 diabetes mellitus patients". Acta Diabetol 49.2 (2012): 137143.

14. Al-Attas OS., et al. "Blood thiamine and its phosphate esters as measured by high-performance liquid chromatography: levels and associations in diabetes mellitus patients with varying degrees of microalbuminuria". Journal of Endocrinological Investigation 35.11 (2012): 951-956.

15. GonzA lez-Ortiz M., et al. "Effect of thiamine administration on metabolic profile, cytokines and inflammatory markers in drug-naA_ve patients with type 2 diabetes". European Journal of Nutrition 2011 50(2):145-149.

16. Hellmann H and Mooney S. "Vitamin B6: a molecule for human health?”. Molecules 15.1 (2010): 442-459.

17. Tavintharan S., et al. "Niacin results in reduced monocyte adhesion in patients with type 2 diabetes mellitus". Atherosclerosis 215.1 (2011): 176-179.

18. Sahin K., et al. "Anti-diabetic activity of chromium picolinate and biotin in rats with type 2 diabetes induced by high-fat diet and streptozotocin". British Journal of Nutrition 110.2 (2013): 197-205.

19. Paolisso G., et al. "Metabolic benefits deriving from chronic vitamin C supplementation in aged non-insulin dependent diabetics". Journal of the American College of Nutrition 14 (1995): 387-392. 
20. Hirani V., et al. "Low levels of 25-hydroxy vitamin D and active 1,25-dihydroxyvitamin $\mathrm{D}$ independently associated with type 2 diabetes mellitus in older Australian men: the Concord Health and Ageing in Men Project". Journal of the American Geriatrics Society 62.9 (2014): 1741-1747.

21. Polidori MC., et al. "Plasma levels of lipophilic antioxidants in very old patients with type 2 diabetes". Diabetes/Metabolism Research and Reviews 16.1 (2000): 15-19.

Volume 3 Issue 10 October 2019

(C) All rights are reserved by $P$ Rajalakshmy. 